\title{
Inverse Modeling of Respiratory System during Noninvasive Ventilation by Maximum Likelihood Estimation
}

\author{
Esra Saatci (EURASIP Member) ${ }^{1}$ and Aydin Akan (EURASIP Member) ${ }^{2}$ \\ ${ }^{1}$ Department of Electronic Engineering, Istanbul Kultur University, Bakirkoy, 34156 Istanbul, Turkey \\ ${ }^{2}$ Department of Electrical and Electronics Engineering, Istanbul University, Avcilar, 34320 Istanbul, Turkey \\ Correspondence should be addressed to Esra Saatci, esra.saatci@iku.edu.tr
}

Received 2 October 2009; Revised 25 February 2010; Accepted 31 May 2010

Academic Editor: Satya Dharanipragada

Copyright ( 92010 E. Saatci and A. Akan. This is an open access article distributed under the Creative Commons Attribution License, which permits unrestricted use, distribution, and reproduction in any medium, provided the original work is properly cited.

\begin{abstract}
We propose a procedure to estimate the model parameters of presented nonlinear Resistance-Capacitance (RC) and the widely used linear Resistance-Inductance-Capacitance (RIC) models of the respiratory system by Maximum Likelihood Estimator (MLE). The measurement noise is assumed to be Generalized Gaussian Distributed (GGD), and the variance and the shape factor of the measurement noise are estimated by MLE and Kurtosis method, respectively. The performance of the MLE algorithm is also demonstrated by the Cramer-Rao Lower Bound (CRLB) with artificially produced respiratory signals. Airway flow, mask pressure, and lung volume are measured from patients with Chronic Obstructive Pulmonary Disease (COPD) under the noninvasive ventilation and from healthy subjects. Simulations show that respiratory signals from healthy subjects are better represented by the RIC model compared to the nonlinear RC model. On the other hand, the Patient group respiratory signals are fitted to the nonlinear RC model with lower measurement noise variance, better converged measurement noise shape factor, and model parameter tracks. Also, it is observed that for the Patient group the shape factor of the measurement noise converges to values between 1 and 2 whereas for the Control group shape factor values are estimated in the super-Gaussian area.
\end{abstract}

\section{Introduction}

The assessment of the respiratory function is an important part of the clinical medicine [1]. Although the clinicians use some standard evaluation techniques and there are bewildering variety of computerized test equipments, the automatic measurement of the lung mechanics requires further work. The main existing problems presented are the following. (i) The lung is a dynamic system such that its parameters should be monitored continuously even with the ventilatory assistance [2]; (ii) the signals measured at the output of this dynamic system, the input, and the system parameters might be nonlinearly related to each other over one breathing period $[2,3]$; and (iii) the proposed methods for investigating the lung mechanics should not require any kind of patient's cooperation.

Using the measured respiratory signals (i.e., airway flow, $\dot{V}(t)$, and airway pressure (mask pressure), $\left.P_{\text {aw }}(t)\right)$, in the literature, conventional least square (LS) and recursive least square methods were used to estimate the linear and nonlinear model parameters of the respiratory system [47]. Regarding the measured time series of airway flow and mask pressure, the abovementioned studies have two major assumptions: (i) the airway flow and the mask pressure are deterministic signals, and (ii) the uncertainty (referred to as a measurement noise) between the measurements and the model is zero-mean Gaussian distributed white noise. However, to the best of our knowledge, there is no study which attempts to define the noise in the respiratory system model fitting to the measured respiratory signals. Thus it is of interest to choose generalized noise model to express the measurement noise involved in the respiratory system identification problem.

In this study, we present the well-known Maximum Likelihood Estimation (MLE) for the respiratory parameter estimation, by assuming that the measurement noise is General Gaussian Distributed (GGD). MLE together with GGD constitute a statistically powerful method which allows more 
degrees of freedom to explore the statistical parameters of the measurement noise. In the simulations, recently presented nonlinear Resistance-Capacitance (RC) and widely used linear Resistance-Inductance-Capacitance (RIC) models [5, 8 ] were used to represent the respiratory system. Accordingly, one of our aims was to derive the theoretical expressions of the presented lung models in the framework of the MLE algorithm with the GGD noise model. In this respect, the artificially produced airway flow and the mask pressure signals that mimic the patients with Chronic Obstructive Pulmonary Disease (COPD) under non-invasive mechanical ventilation were used for the estimator assessment. Then, the parameters of both respiratory models were estimated from the observed signals collected from the COPD patients under non-invasive mechanical ventilation (Patient group) and the healthy subjects (Control group).

The rest of the paper is organized as follows. Section 2 reviews the nonlinear RC and RIC models of the respiratory system during non-invasive ventilation. The measurement equations of the models are also drawn in Section 2. In Section 3, MLE and GGD are summarized and the estimation of the RIC and nonlinear RC models' parameters is explained. Also estimator performance assessment criteria (i.e., the Cramer-RAO Lower Bound (CRLB)) were depicted in the same section. In Section 4, the experimental procedure was explained. The results of the simulations are presented and discussed in Section 5. Finally, in Section 6 conclusions are drawn.

\section{Respiratory Models}

In this paper we used the nonlinear RC [8] and RIC models [5] of the respiratory system. The nonlinear RC model is the simplified version of the lung model in [3] where its parameters are also verified. Since, time-domain methods require well description of the respiratory system, the non-invasive ventilation and muscular pressure effects should be included to both models for the complete system representation. $P_{\text {mus }}(t)$ represents the pressure effects on the measured $P_{\mathrm{aw}}(t)$ produced by the patient's inspiratory muscles. The ventilator-generated pressure $P_{\text {ven }}(t)$ has a direct effect on $P_{\mathrm{aw}}(t)$ as it is the major positive component shaping the waveform. To express the reality, $P_{\mathrm{ven}}(t)$ is only used in models of the Patient Group. It should be emphasized that pressure sources $P_{\text {mus }}(t)$ and $P_{\text {ven }}(t)$ reflect only the related effects on the $P_{\mathrm{aw}}(t)$; thus they should not be considered as direct lung model functions. $P_{\text {mus }}(t)$ can be approximated by the second-order polynomial function [9]:

$$
P_{\text {mus }}(t)= \begin{cases}-P_{\text {mus max }}\left(1-\frac{t}{T_{I}}\right)^{2}+P_{\text {mus max }}, & 0 \leq t \leq T_{I}, \\ P_{\text {mus max }} e^{-t / \tau_{m}}, & T_{I} \leq t \leq T,\end{cases}
$$

where $P_{\text {mus max }}$ represents the effect of the maximal patient's effort on $P_{\mathrm{aw}}(t), T_{I}$ and $T$ are the inspiration duration and the total duration of one respiration cycle, respectively. In this paper $P_{\text {mus max }}$ is added to the unknown parameter vector whereas $T_{I}$ and $T$ are set to constant values derived

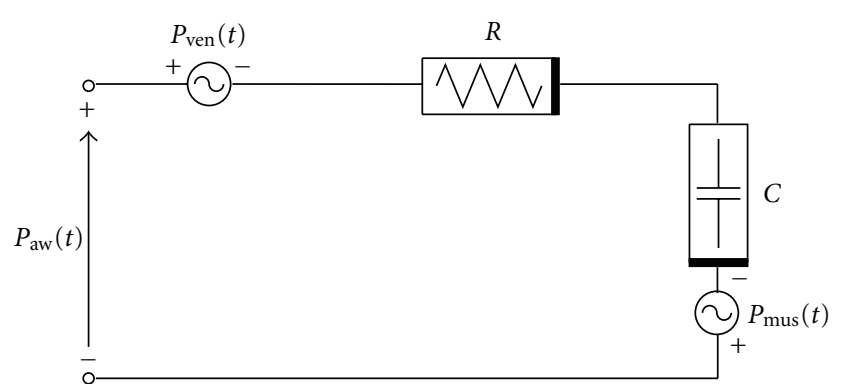

(a)

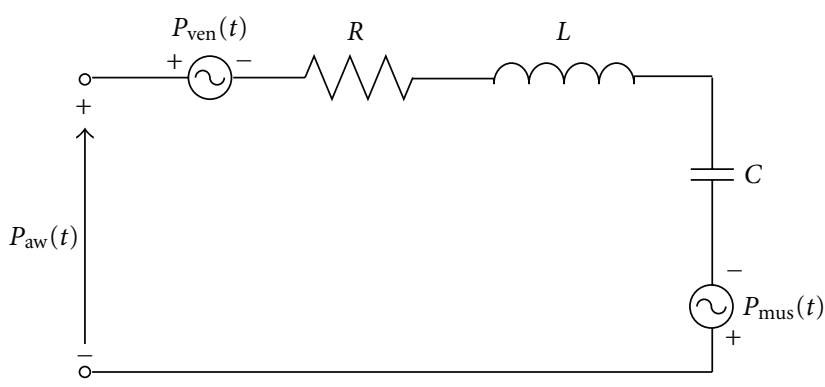

(b)

FIGURE 1: (a) Nonlinear one-compartment respiration model with noninvasive ventilator effect. (b) Linear one-compartment RIC model of the lung with noninvasive ventilator effect.

from the experimental signals. The time constant $\tau_{m}$ is an important parameter for mostly determining the expiratory asynchrony in the noninvasive ventilation [9]. A constant value was chosen for $\tau_{m}$ in order to resemble the respiratory system.

The ventilator-generated pressure $P_{\text {ven }}$ is modeled by the exponential function [9]:

$$
P_{\mathrm{ven}}(t)= \begin{cases}\text { PEEP, } & 0 \leq t \leq t_{\text {trig }}, \\ P_{\mathrm{ps}}\left(1-e^{-t / \tau_{\mathrm{vi}}}\right), & t_{\text {trig }}<t \leq T_{I}, \\ P_{\mathrm{ps}}\left(e^{-t / \tau_{\mathrm{ve}}}\right), & T_{I}<t \leq T,\end{cases}
$$

where $P_{\mathrm{ps}}$ and PEEP represent the maximal ventilation pressure and Positive End Expiration Pressure, respectively. The ventilator inspiration time constant $\tau_{\mathrm{vi}}$ corresponds to the flow acceleration speed of the ventilator, whereas the ventilator expiration time constant $\tau_{\mathrm{ve}}$ is the ventilator deceleration speed and contributes to the pressure rise at the termination of the inspiration. The set values for the parameters in $P_{\text {mus }}(t)$ and $P_{\text {ven }}(t)$ are in Table $1 . P_{\text {mus }}(t)$ and $P_{\text {ven }}(t)$ were applied in the simulations of the artificial signals and COPD patients' signals, whereas, for the control group, $P_{\text {ven }}(t)$ was not included to respiratory models.

Figure 1 shows the nonlinear RC and RIC models of the respiratory system. In continuous time, the model output 
equations, respectively, are

$$
\begin{aligned}
P_{\mathrm{aw}}(t)= & \left(A_{u}+K_{u}|\dot{V}(t)|\right) \dot{V}(t)+A_{l} e^{K_{l} V(t)} \\
& +B_{l}-P_{\mathrm{mus}}(t)+P_{\mathrm{ven}}(t), \\
P_{\mathrm{aw}}(t)= & V(t) E+\dot{V}(t) R+\ddot{V}(t) L \\
& -P_{\mathrm{mus}}(t)+P_{\mathrm{ven}}(t),
\end{aligned}
$$

where $A_{u}, K_{u}, A_{l}, K_{l}, B_{l}$, and $R, E, L$ constitute the unknown parameter vectors for the nonlinear RC and RIC models, respectively. $P_{\mathrm{aw}}(t)$ is the measured mask pressure and $\dot{V}(t)$ is the measured airway flow. $V(t)$ represents the gas volume changes above the Residual Volume (RV) in the lungs and $\ddot{V}(t)$ is the gradient of the airway flow.

In discrete form, the observed time series of mask pressure for the nonlinear RC and RIC models of the respiratory system are given by

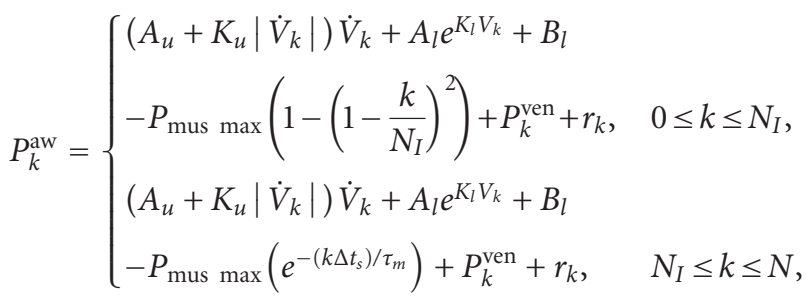

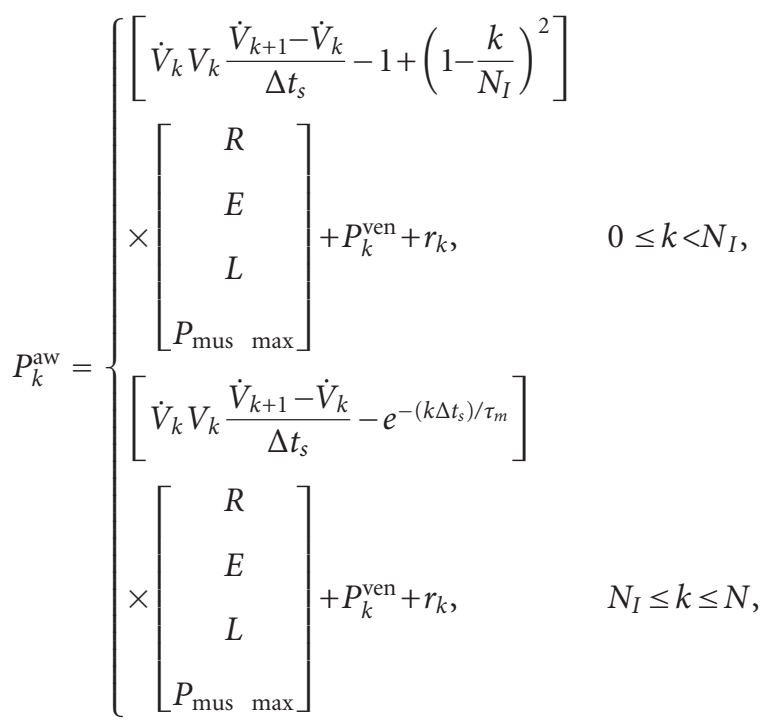

where $r_{k}$ is the GGD measurement noise; $N$ and $N_{I}$ are the discrete versions of $T$ and $T_{I}$; discrete time steps are

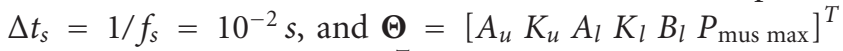
and $\Theta=\left[\begin{array}{llll}R & L & E & P_{\text {mus max }}\end{array}\right]^{T}$ are the parameter vectors to be estimated.

Deriving (4) and (5) constitutes the first step for the respiratory parameter estimation by MLE.

\section{Estimation of the Model Parameters by MLE}

If the nonlinear time series model $\mathbf{y}_{k}=\mathrm{h}_{k}\left(\mathbf{x}_{k}, \boldsymbol{\Theta}, \mathbf{u}_{k}, \mathbf{r}_{k}\right)$ is considered, then MLE algorithm can be applied to estimate the unknown parameters, $\boldsymbol{\Theta}$, where $\mathbf{x}_{k}$ are the model states, $\mathbf{u}_{k}$ are known inputs, and $\mathbf{r}_{k}$ represents the measurement noise. Since the states are measured signals in our models, we omit $\mathbf{x}_{k}$ terms in the MLE derivations. In MLE, the parameters are assumed to be the unknown but deterministic constants and the measurements are used to calculate the likelihood as a function of the parameters. Then the parameters are calculated by

$$
\frac{\delta \log \left(L\left(\boldsymbol{\Theta} \mid \mathbf{y}_{1: N}\right)\right)}{\delta \Theta_{m}}=0, \quad m=1, \ldots, p,
$$

where $p$ is the total number of the parameters. In this study, the Newton-Raphson (NR) method is used for the iterative maximization of (6). At the $(i+1)$ th iteration, the parameter vector is computed by

$$
\boldsymbol{\Theta}_{(i+1)}=\boldsymbol{\Theta}_{(i)}-\mathbf{g}\left(\boldsymbol{\Theta}_{(i)}\right) \mathbf{H}\left(\boldsymbol{\Theta}_{(i)}\right)^{-1},
$$

where the indices represented in the parentheses are the iteration steps. $\mathbf{g}(\cdot)$ function denotes the gradient vector of the logarithmic likelihood function, whereas $\mathbf{H}(\cdot)$ is the Hessian matrix, composed of the second deviates of the logarithmic likelihood function:

$$
\begin{aligned}
& \mathbf{g}(\boldsymbol{\Theta})=\left[\frac{\partial \log \left(L\left(\mathbf{y}_{1: k} ; \boldsymbol{\Theta}\right)\right)}{\partial \Theta_{m}}\right]_{1, m}, \\
& \mathbf{H}(\boldsymbol{\Theta})=\left[\frac{\partial^{2} \log \left(L\left(\mathbf{y}_{1: k} ; \boldsymbol{\Theta}\right)\right)}{\partial \Theta_{m} \partial \Theta_{r}}\right]_{m, r} .
\end{aligned}
$$

Iteration of (7) continues until the condition for the set $\mathcal{E}, L\left(\widehat{\boldsymbol{\Theta}}_{M L} \mid \mathbf{y}_{1: k}\right)-L\left(\boldsymbol{\Theta} \mid \mathbf{y}_{1: k}\right) \geq \varepsilon$ or $i=i_{\max }$, is met. Then, the final iteration provides the parameter vector, $\Theta$. Moreover, the estimator variance is calculated as

$$
\operatorname{var}(\widehat{\Theta})=\mathbf{H}(\boldsymbol{\Theta})^{-1}
$$

We now need to define the measurement noise and propose the probability distribution for the best convergence of the parameters.

3.1. Generalized Gaussian Distributed Noise Model. Measurement noise has two main components: (i) the noiselike residuals resulted from the model fitting to the measured signals (biggest part); (ii) random noise resulted from the measurement equipment and the valve system of the noninvasive ventilator. Based on the assumption that measurement noise, $\mathbf{r}_{k}$, is GGD type, the measurement probability distribution can be given as

$$
\operatorname{Pr}\left(\mathbf{y}_{1: k} ; \boldsymbol{\Theta}\right)=c \exp \left\{-\frac{1}{A\left(p_{r}, \sigma_{r}\right)^{p_{r}}} \sum_{k=1}^{N}\left|y_{k}-h_{k}(\boldsymbol{\Theta})\right|^{p_{r}}\right\},
$$



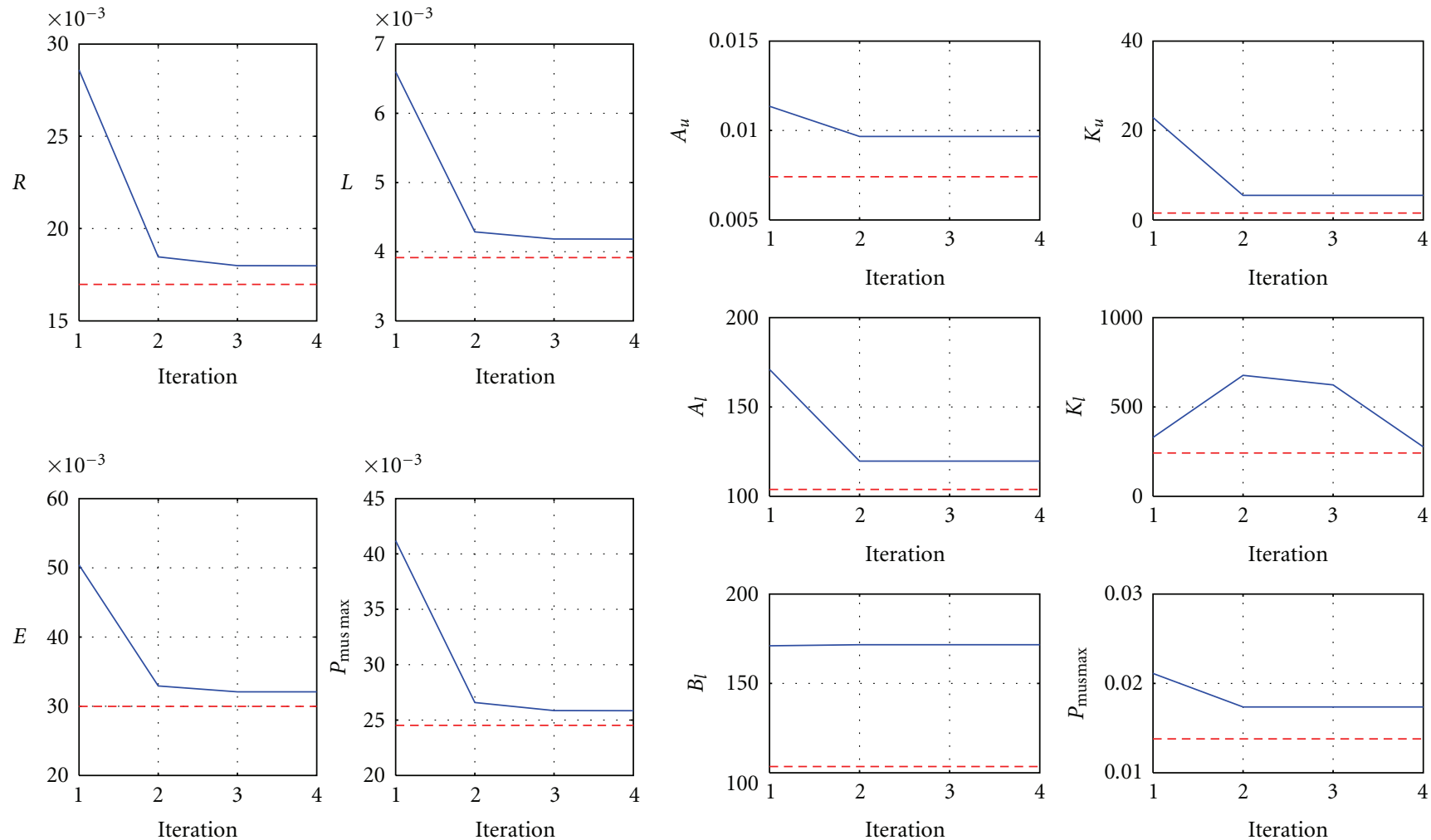

(a)
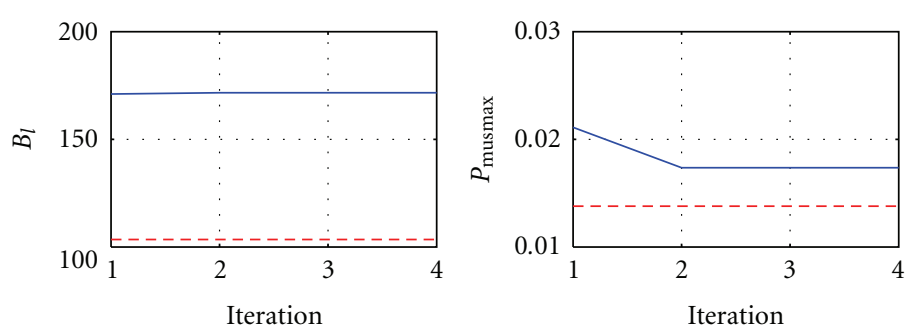

(b)

Figure 2: Cramer-Rao Lower Bounds (dotted red line) and estimator variances (black line) versus iteration.
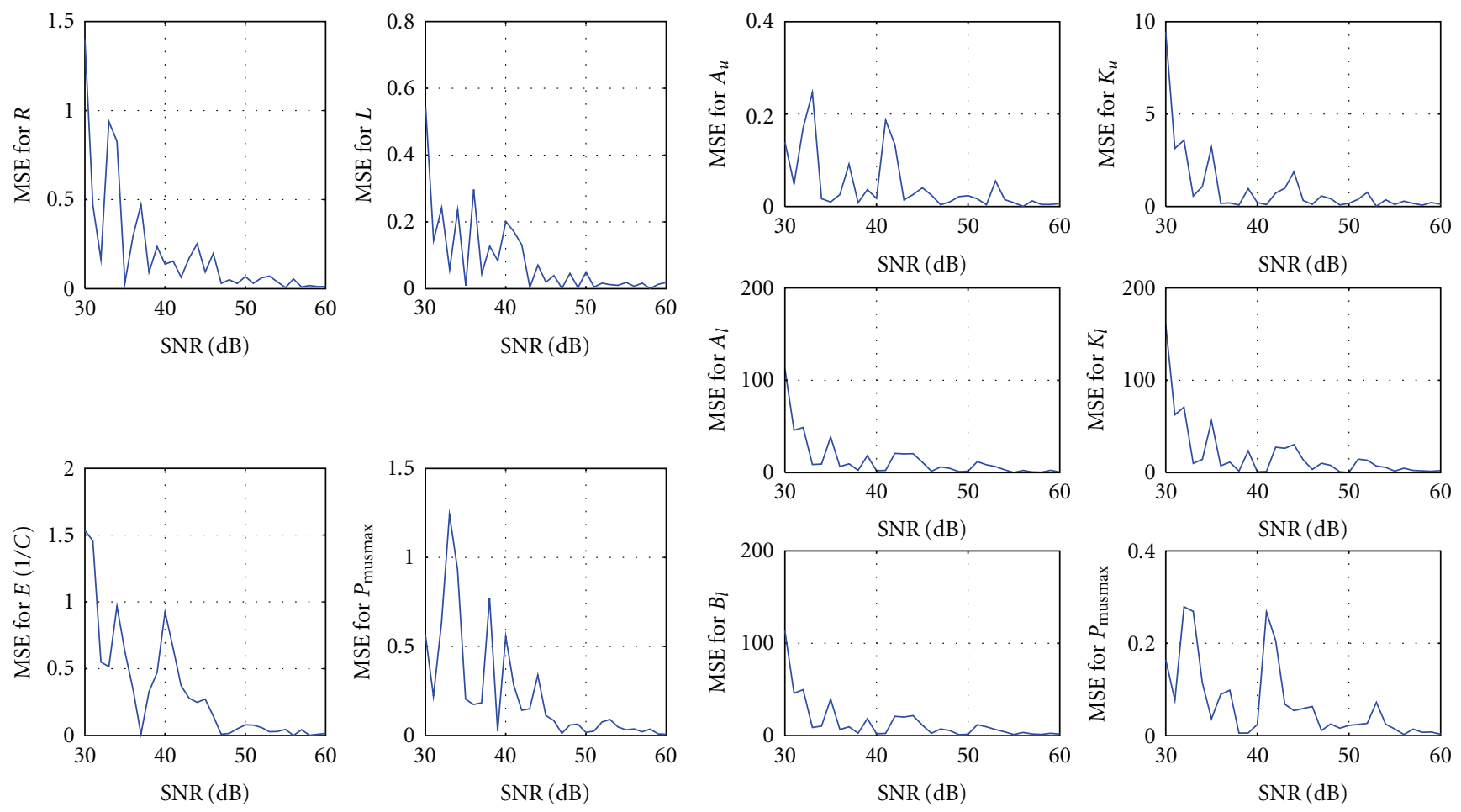

(a)
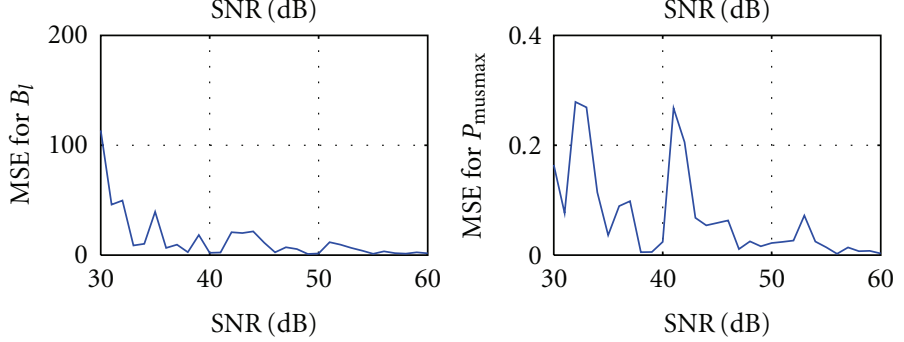

(b)

FIGURE 3: (a) Mean Squared Error (MSE) versus SNR for linear RIC model parameter estimation produced by artificial signals. (b) Mean Squared Error (MSE) versus SNR for nonlinear model parameter estimation produced by artificial signals. 
where $h_{k}(\boldsymbol{\Theta})$ is the many-to-one function that yields the left side of (4) or (5). $c$ can be defined as $c=$ $1 /\left(2^{N} \Gamma\left(1+1 / p_{r}\right)^{N} A\left(p_{r}, \sigma_{r}\right)^{N}\right)$, where $\Gamma(\cdot)$ is the Gamma function and $A\left(p_{r}, \sigma_{r}\right)=\left[\sigma_{r}^{2} \Gamma\left(1 / p_{r}\right) / \Gamma\left(3 / p_{r}\right)\right]^{1 / 2}$ is a scaling factor which allows that $\operatorname{var}\left(r_{k}\right)=\sigma_{r}^{2}$. The variance and the shape factor of GGD are represented as $\sigma_{r}^{2}$ and $p_{r}$, respectively. These two parameters should be estimated in order to define the measurement noise. It is reminded that since the respiratory system, consequently respiratory signals, differs between diseased and healthy cases, we expect the parameters of GGD-based measurement noise to provide the valuable information about the respiratory models fit to the measured signals.

3.1.1. Measurement Noise Variance Estimation. A common way of estimating the measurement noise variance consists of maximizing the logarithmic likelihood function with respect to $\sigma_{r}^{2}$ :

$$
\frac{\partial \log \left(L\left(\mathbf{y}_{1: N} ; \Theta, \sigma_{r}, p_{r}\right)\right)}{\partial \sigma_{r}}=0 .
$$

With the help of the conditional probability theorem, by (10), the logarithmic likelihood function and the variance in the closed form can be obtained, respectively, as

$$
\begin{aligned}
L\left(\mathbf{y}_{1: k} ; \theta\right)= & -N\left(\log 2+\log \Gamma\left(1+\left(\frac{1}{p_{r}}\right)\right)+\log A\left(p_{r}, \sigma_{r}\right)\right) \\
& -\frac{1}{A\left(p_{r}, \sigma_{r}\right)^{p_{r}}} \sum_{k=1}^{N}\left|y_{k}-h_{k}(\boldsymbol{\Theta})\right|^{p_{r}},
\end{aligned}
$$$$
\widehat{\sigma}_{r}=\left[\frac{p_{r(i)}}{N}\left[\frac{\Gamma\left(1 / p_{r(i)}\right)}{\Gamma\left(3 / p_{r(i)}\right)}\right]^{-p_{r(i)} / 2} \sum_{k=1}^{N}\left|y_{k}-h_{k}\left(\Theta_{(i)}\right)\right|^{p_{r(i)}}\right]^{1 / p_{r(i)}} .
$$

It should also be noted that, in the above derivation, the system model parameters, $\boldsymbol{\Theta}$, and the measurement noise shape factor, $p_{r}$, are considered as known quantities.

3.1.2. Measurement Noise Shape Factor Estimation. In the GGD models the shape factor, $p_{r}$, can be estimated with four different methods [10]. In this study, the Kurtosis ratio method was chosen due to its simplicity and its success for the small values of $p_{r}$. The Kurtosis ratio is computed by

$$
\xi\left(\hat{p}_{r}\right)=\frac{E\left[\left|r_{k}\right|^{2}\right]}{\sqrt{E\left[\left|r_{k}\right|^{4}\right]}}=\frac{\Gamma\left(3 / \hat{p}_{r}\right)}{\sqrt{\Gamma\left(5 / \hat{p}_{r}\right) \Gamma\left(1 / \hat{p}_{r}\right)}},
$$

where $E\left[\left|r_{k}\right|^{4}\right]$ and $E\left[\left|r_{k}\right|^{2}\right]$ denote the fourth- and secondorder central moments of the measurement noise, respectively. However, (14) cannot be solved in the explicit form. Thus, a lookup table was generated for the inversion of the Kurtosis equation.
3.2. Estimation of the Model Parameters. Once the variance and the shape factor of the measurement noise are estimated, parameters of the respiratory models can be computed with the MLE algorithm. Maximization of (12) with respect of $\Theta$ is computed with the Newton-Raphson (NR) algorithm. If $\nabla_{\Theta}$ shows the gradient vector, then the $m$ th element is found as

$$
\nabla_{\theta_{m}} L(\boldsymbol{\Theta})=\frac{1}{A\left(p_{\mathrm{r}}, \sigma_{r}\right)^{p_{r}}} \sum_{k=1}^{N} p_{r}\left|y_{k}-h_{k}(\boldsymbol{\Theta})\right|^{p_{r}-1} \frac{\partial h_{k}(\boldsymbol{\Theta})}{\partial \boldsymbol{\Theta}_{m}},
$$

and from (12) the Hessian matrix can be achieved by

$$
\begin{aligned}
& \nabla_{\theta_{m}} \nabla_{\theta_{r}} L(\boldsymbol{\Theta}) \\
& =\frac{p_{r}}{A\left(p_{r}, \sigma_{r}\right)} \sum_{k=1}^{N}\left\{-\left(p_{r}-1\right)\left|y_{k}-h_{k}(\boldsymbol{\Theta})\right|^{p_{r} 2} \frac{\partial h_{k}(\boldsymbol{\Theta})}{\partial \theta_{m}} \frac{\partial h_{k}(\boldsymbol{\Theta})}{\partial \theta_{r}}\right. \\
& \left.+\left|y_{k}-h_{k}(\boldsymbol{\Theta})\right|^{p_{r}-1} \frac{\partial^{2} h_{k}(\boldsymbol{\Theta})}{\partial \theta_{m} \partial \theta_{r}}\right\},
\end{aligned}
$$

where $\left|y_{k}-h_{k}(\boldsymbol{\Theta})\right|$ is the error and the second term in the sum can be ignored with respect to the first term; thus $(m, r)$ th element of the Hessian matrix becomes

$$
\begin{aligned}
& \nabla_{\theta_{m}} \nabla_{\theta_{r}} L(\boldsymbol{\Theta}) \\
& =\frac{-p_{r}\left(p_{r}-1\right)}{A\left(p_{r}, \sigma_{\mathrm{r}}\right)} \sum_{k=1}^{N}\left\{\left|y_{k}-h_{k}(\boldsymbol{\Theta})\right|^{p_{r}-2} \frac{\partial h_{k}(\boldsymbol{\Theta})}{\partial \theta_{m}} \frac{\partial h_{k}(\boldsymbol{\Theta})}{\partial \theta_{r}}\right\} .
\end{aligned}
$$

Equations (15), (17), and (7) form the basis of the MLE algorithm. It should also be noted that in the above derivation the measurement noise shape factor, $p_{r}$, and variance, $\sigma_{r}^{2}$, are considered as known quantities.

3.3. Estimator Performance Measurement. The MLE algorithm, shown in Algorithm 1, was coded in Matlab and applied first to the artificially generated respiratory signals (mask pressure, airway flow, and lung volume), then to respiratory signals acquired from the COPD patients with the noninvasive ventilator assistance, and finally to the healthy subjects. Before depicting the results from the real respiratory signals, the performance of the estimator which is bounded by the respiratory models and the properties of the respiratory signals should be demonstrated. In statistical signal processing there are two ways to measure the estimator's ability: (i) computing the Lower Bounds (LBs) on the estimator's variance and (ii) calculating the Mean Square Error (MSE) on the estimated parameters. Both LB and MSE are calculated with the true value of the parameters, thus with the artificial signals. In the case of real signals, we estimated the parameters from five different breath cycles of the same subject and plotted on the same figure. Apart from the effects of the small differences between breath cycles, the estimator is expected to result in the same parameter estimations. This procedure acts as a self-performance measurement in the real signal processing. 


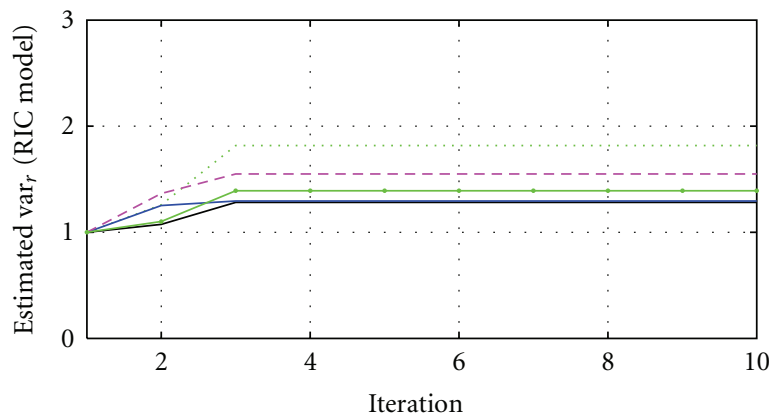

(a)

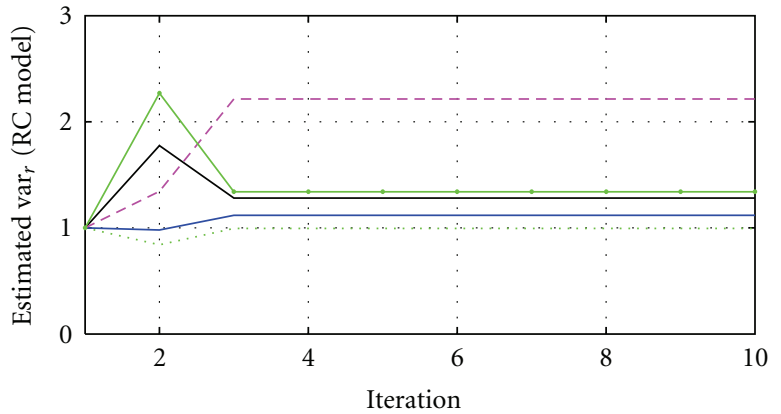

(b)

FIGURE 4: Estimated measurement noise variance produced by representative COPD patient (a) for RIC model and (b) for nonlinear RC model.

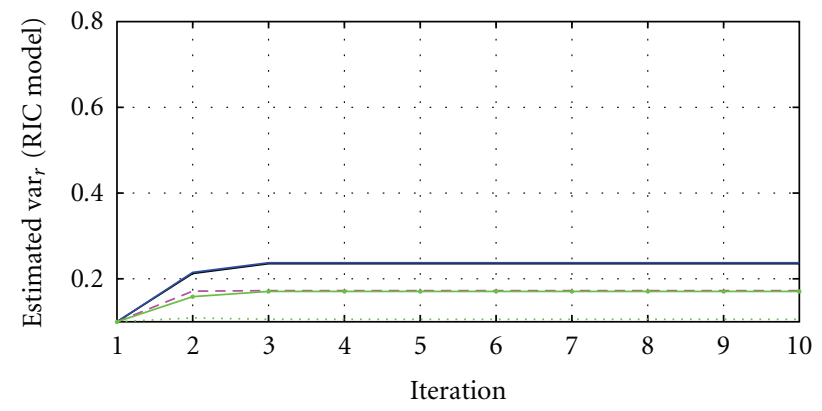

(a)

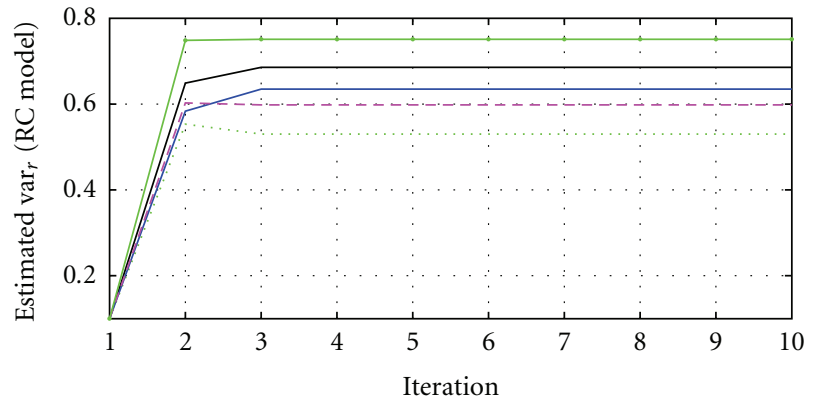

(b)

FIGURE 5: Estimated measurement noise variance produced by representative healthy patient (a) for RIC model and (b) for nonlinear RC model.

3.3.1. Artificial Respiratory Signal. The airway flow inside the upper airways was simulated as a sinusoidal signal with the parameters shown in Table 1. The lung volume (i.e., Tidal Volume) was calculated by the integration of the airway flow over one breathing cycle. Pressure inside the facemask was calculated by (14) for the nonlinear RC model and by (12) for the RIC model with the help of the model parameters shown in Table 1. Zero-mean white Gaussian noise with different SNR levels was then added to the airway pressure and airway flow.

3.3.2. Initial Values. Since Maximum Likelihood incorporates the "maximization" step and uses hill-climbing approach, reaching only to local minima can be guaranteed. However, there are two ways to avoid local minima: (1) to change the initial values for different starting points and (2) to find the optimum initial values by considering a nonlinear respiratory model and the constraints on the parameters. In our studies we employed the two approaches. Monte Carlo averaging $(M C=100)$ is used to decrease the effects of the initial value selection whereas initial values are generated from the constrained sets of the parameters. For the real data case, same initial values were used for five different breath cycle simulations. The mean and variance of the initial values were chosen solely to achieve the convergence of the parameters. On the other hand, the initial values of $\sigma_{r}^{2}$ and $p_{r}$ were set to constant values. Table 2 summarizes the initial values selected for the simulations.

3.3.3. Cramer-Rao Lower Bounds. Cramer-Rao Lower Bound (CRLB) [11] is defined as

$$
\mathrm{CRB}(\boldsymbol{\Theta})=\mathbf{I}(\boldsymbol{\Theta})^{-1},
$$

where $\mathbf{I}(\boldsymbol{\Theta})=-E\left\{\left(\partial^{2} L\left(\mathbf{y}_{1: k} ; \boldsymbol{\Theta}\right)\right) /\left(\partial \Theta_{m} \partial \Theta_{r}\right)\right\}$ is called as the Fisher Information matrix. If $p_{r}=2$ and $p_{r}=1$ (i.e., the measurement noise is considered as Gaussian and Laplacian distributed), then the expected value of (16) with respect to the parameters vector yields, respectively, that

$$
\begin{gathered}
\mathbf{I}_{p_{r}=2}(\boldsymbol{\Theta})=\frac{2}{A\left(2, \sigma_{r}\right)^{2}} \sum_{k=1}^{N} \frac{\partial h_{k}(\boldsymbol{\Theta})}{\partial \boldsymbol{\Theta}_{m}} \frac{\partial h_{k}(\boldsymbol{\Theta})}{\partial \boldsymbol{\Theta}_{r}} \\
\mathbf{I}_{p_{r}=1}(\boldsymbol{\Theta})=-\frac{1}{A\left(2, \sigma_{r}\right)^{2}} \sum_{k=1}^{N} \frac{\partial^{2} h_{k}(\boldsymbol{\Theta})}{\partial \theta_{m} \partial \theta_{r}}
\end{gathered}
$$

Pham and DeFigueiredo [12] showed that ML estimate of the mean of GGD noise is unbiased for $1 \leq p_{r}<\infty$. Moreover, the asymptotic behavior of ML estimates for the uniform and Laplacian noises has been investigated in [13] yielding that, even if the priori distribution of a noise is unknown, the stochastic CRB can be attained asymptotically by the ML estimator derived for a Gaussian signal. Hence, 


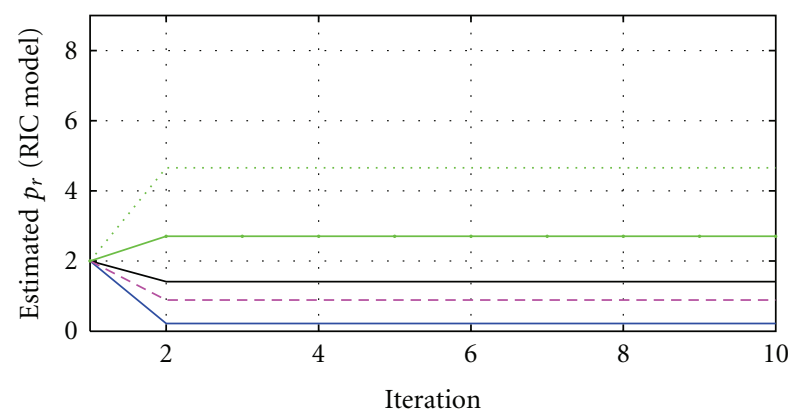

(a)

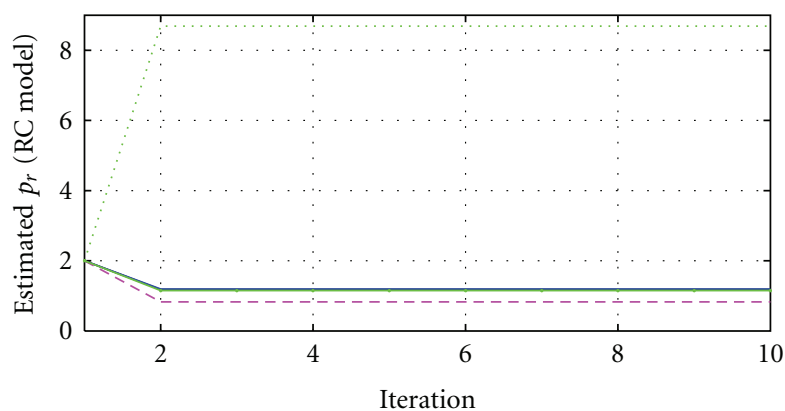

(b)

FIGURE 6: Estimated measurement noise shape factor produced by representative COPD patient (a) for RIC model and (b) for nonlinear RC model.

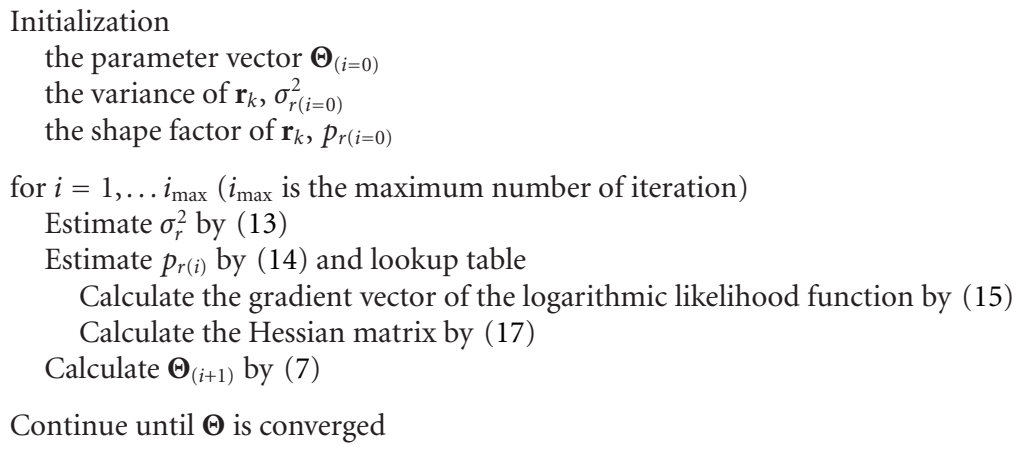

Algorithm 1: MLE Algorithm.

TABLE 1: Parameters of the respiratory signals in the simulations. ER and EMP stand for experimental reading and estimated model parameter, respectively.

\begin{tabular}{|c|c|c|c|c|}
\hline \multirow[t]{2}{*}{ Model } & \multirow[t]{2}{*}{ Parameter } & \multicolumn{3}{|c|}{ Value } \\
\hline & & Artificial Sig. & Patient G. & Control G. \\
\hline Noninvasive ventilator & PEEP & $4 \mathrm{~cm} \mathrm{H}_{2} \mathrm{O}$ & ER & N/A \\
\hline \multirow[t]{7}{*}{ Pressure simulation } & $P_{\mathrm{ps}}$ & $6 \mathrm{~cm} \mathrm{H}_{2} \mathrm{O}$ & ER & N/A \\
\hline & $\tau_{\mathrm{vi}}$ & $0.006 s$ & $0.006 s$ & N/A \\
\hline & $\tau_{\mathrm{ve}}$ & $0.006 s$ & $0.006 s$ & N/A \\
\hline & $k_{\text {trig }}\left(t_{\text {trig }}\right)$ & $N / 10(T / 10)$ & ER & N/A \\
\hline & $N_{I}\left(T_{I}\right)$ & $N / 3(T / 3)$ & ER & ER \\
\hline & $N(T)$ & $300(3 s)$ & ER & ER \\
\hline & $f_{s}$ & $100 \mathrm{~Hz}$ & $100 \mathrm{~Hz}$ & $100 \mathrm{~Hz}$ \\
\hline Muscular & $P_{\text {mus max }}$ & $1 \mathrm{~cm} \mathrm{H}_{2} \mathrm{O}$ & EMP & EMP \\
\hline Pressure simulation & $\tau_{m}$ & $60 \mathrm{~ms}$ & $60 \mathrm{~ms}$ & $60 \mathrm{~ms}$ \\
\hline Nonlinear respiration & $A_{u}$ & $3.1 \mathrm{~cm} \mathrm{H}_{2} \mathrm{O} \cdot \mathrm{s} / \mathrm{l}$ & EMP & EMP \\
\hline Model parameters & $K_{u}$ & $0.32 \mathrm{~cm} \mathrm{H}_{2} \mathrm{O} \cdot s^{2} / l^{2}$ & EMP & EMP \\
\hline \multirow[t]{4}{*}{$\Theta$} & $A_{l}$ & $0.1 \mathrm{~cm} \mathrm{H}_{2} \mathrm{O}$ & EMP & EMP \\
\hline & $K_{l}$ & 1.0 & EMP & EMP \\
\hline & $B_{l}$ & $0 \mathrm{~cm} \mathrm{H}_{2} \mathrm{O}$ & EMP & EMP \\
\hline & $P_{\text {mus max }}$ & $1 \mathrm{~cm} \mathrm{H}_{2} \mathrm{O}$ & EMP & EMP \\
\hline Linear RIC & $R$ & $2.75 \mathrm{~cm} \mathrm{H}_{2} \mathrm{O} \cdot \mathrm{s} / \mathrm{l}$ & EMP & EMP \\
\hline Model parameters & $L$ & $0.0063 \mathrm{~cm} \mathrm{H}_{2} \mathrm{O} \cdot s^{2} / l$ & EMP & EMP \\
\hline$\Theta$ & $E=1 / C$ & $85.8 \mathrm{~cm} \mathrm{H}_{2} \mathrm{O} / \mathrm{l}$ & EMP & EMP \\
\hline
\end{tabular}


TABLE 2: Initial values selected for the simulations. $\mathcal{N}$ stands for the Gaussian distribution.

\begin{tabular}{|c|c|c|c|}
\hline \multirow[t]{2}{*}{ Parameter } & \multicolumn{3}{|c|}{ Value } \\
\hline & Artificial Sig. & Patient G. & Control G. \\
\hline & \multicolumn{3}{|c|}{ RIC model } \\
\hline Parameter vector $\boldsymbol{\Theta}_{(i=0)}$ & $\mathcal{N}(1 / 2,1 / 4)$ & $\mathcal{N}(1 / 2,1 / 4)$ & $\mathcal{N}(1 / 2,1 / 4)$ \\
\hline Variance of $\mathbf{r}_{k}, \sigma_{r(i=0)}^{2}$ & 0.1 & 1 & 0.1 \\
\hline \multirow[t]{2}{*}{ Shape factor of $\mathbf{r}_{k}, p_{r(i=0)}$} & 2 & 2 & 2 \\
\hline & \multicolumn{3}{|c|}{ Nonlinear RC model } \\
\hline Parameter vector $\boldsymbol{\Theta}_{(i=0)}$ & $\mathcal{N}(1 / 2,1 / 4)$ & $\mathcal{N}(5,25)$ & $\mathcal{N}(1,1)$ \\
\hline Variance of $\mathbf{r}_{k}, \sigma_{r(i=0)}^{2}$ & 0.1 & 1 & 0.1 \\
\hline Shape factor of $\mathbf{r}_{k}, p_{r(i=0)}$ & 2 & 2 & 2 \\
\hline
\end{tabular}

for the GGD-type measurement noise having shape factors other than $p_{r}=1,2$, we use asymptotic property of MLE and accept (19) for the bound calculation.

Figure 2 shows the CRLB for the parameter estimates together with the estimator variances in one breath cycle. First of all, although we have convergence of the parameters at $i=2$, we set $i_{\max }=4$ for the demonstration of the lower bound clearly. CRLB and parameter error variances are calculated by (19) and (9), respectively, by processing the artificial signals. As it is seen from Figure 2, the variance of the estimator follows the CRLB very closely for all parameters. Especially in RIC model MLE is very successful, because estimator variances reach to the CRLB value at $i=2$ for all parameters. However, for the nonlinear RC model it can be seen that $A_{l}, K_{l}$, and $B_{l}$ have very large CRLB values, whereas $A_{u}$ and $P_{\text {mus max }}$ have lower CRLB values than they have in RIC model. Interestingly, we also observe that estimator variances follow the CRLB for the nonlinear RC model parameters.

We can claim with some confidence that presented CRLB values are the minimum attainable estimator variance bound for the nonlinear RC model. First, CRLB is computed by the real values of the model parameters (no initialization problem), and second, for the GGD-type measurement noise, asymptotic efficiency and lack of bias of the ML estimates can be used (Gaussian noise case). However, we note that, because the Hessian matrix depends on the nonlinear model parameters as well as GGD noise parameters, maximization of the Fisher Information Matrix constitutes high variance bounds. Thus we do not claim that the ML estimators are the best choice among the estimator family. On the other hand, both MSE and CRLB curves clearly suggest that, for the nonlinear RC model, ML estimator gives the optimum variance with regard to the CRLB.

3.3.4. Mean Square Error. The second way to show the estimator performance is to demonstrate the error between the set and the estimated parameters. Mean Square Error (MSE) is calculated by Monte Carlo simulations with $M C=$ 100 as

$$
\operatorname{mse}(\hat{\boldsymbol{\Theta}})=\sqrt{\frac{1}{M C} \sum_{i=1}^{M C}\left(\boldsymbol{\Theta}-\hat{\boldsymbol{\Theta}}_{(i)}\right)^{2}}
$$

and is plotted versus signal-to-noise ratio (SNR). The only reason to use different SNR is to demonstrate the estimator's ability in estimating the parameters' embedded different noise variances. Since the measurement noise consists of the residuals between the real measured signal and the used respiratory model, we expect to estimate various measurement noise variances.

Figure 3 shows the MSE versus SNR plots where Gaussian distributed measurement noise is added. Figure 3 is very inline with Figure 2 in a way that $A_{l}, K_{l}$, and $B_{l}$ parameters show higher MSE values than the rest. This can be easily explained by the nonlinear dependance between these parameters and the airway pressure. In other words, in the nonlinear RC model, the Hessian matrix is calculated as a function of these nonlinear parameters which in turn increases the estimation errors at each iteration. However, although MSE values were higher for nonlinear parameters, the convergence was achieved for all parameters in the nonlinear RC model. It is also interesting to notice that, as it is in CRLB results, $A_{u}$ and $P_{\text {mus max }}$ have lower MSE values than they have in RIC model. That is, although the nonlinear RC model has nonlinear parameters that have relatively higher MSE values, it is still optimum in the sense of estimator performance.

\section{Experiments}

Seven male and one female patients with COPD and four male and two female healthy nonsmoking subjects (without any respiratory disease) were recruited. Patients were breathing with the support of a non-invasive ventilator (Respironics, Inc., BIPAP S/T IPAP: $10-12 \mathrm{~cm} \mathrm{H}_{2} \mathrm{O}$; PEEP: 4-6 $\mathrm{cmH}_{2} \mathrm{O}$ ) via facemask (Respironics, Inc., spectrum size: medium and small), whereas subjects from Control Group were breathing via facemask without the support of the non-invasive ventilation. In both Groups, the mask pressure, the airway flow, and tidal volume were measured by the pneumotachograph and the pressure transducer system (Hans Rudolph, Inc., Research pneumotachograph system). Acquired signals are digitized by a sampling rate of $100 \mathrm{~Hz}$. During acquisition, subjects were awake and in supine position breathing through the facemask. At least 10 breathing cycles of the airway flow, the mask pressure, and the lung volume signals were recorded by the data acquisition 


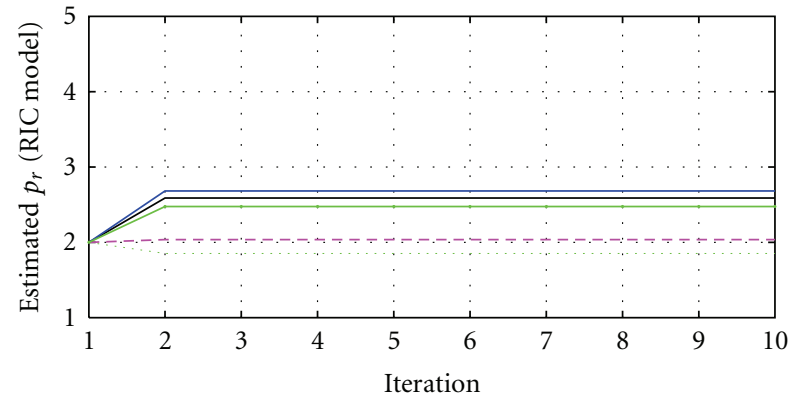

(a)

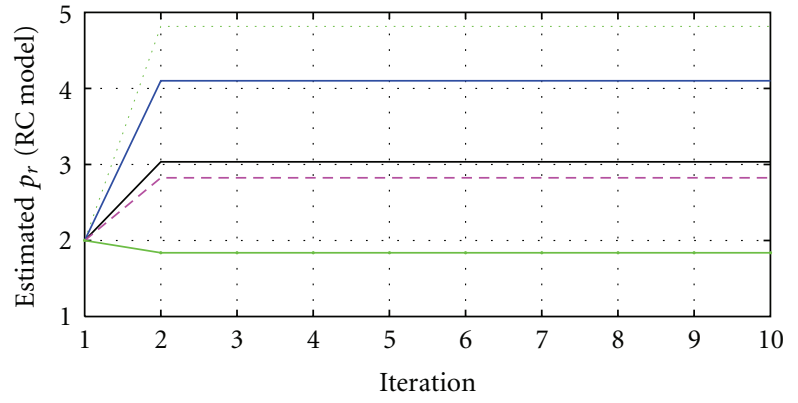

(b)

FIGURE 7: Estimated measurement noise shape factor produced by representative healthy patient (a) for RIC model (b) for nonlinear RC model.
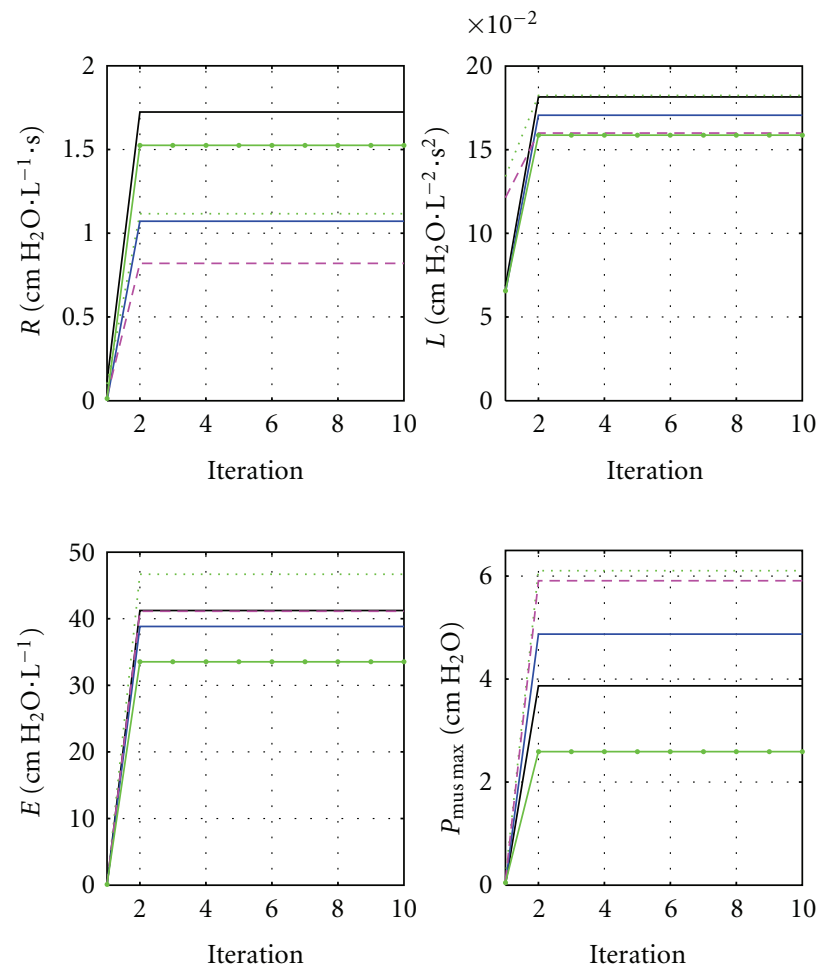

(a)
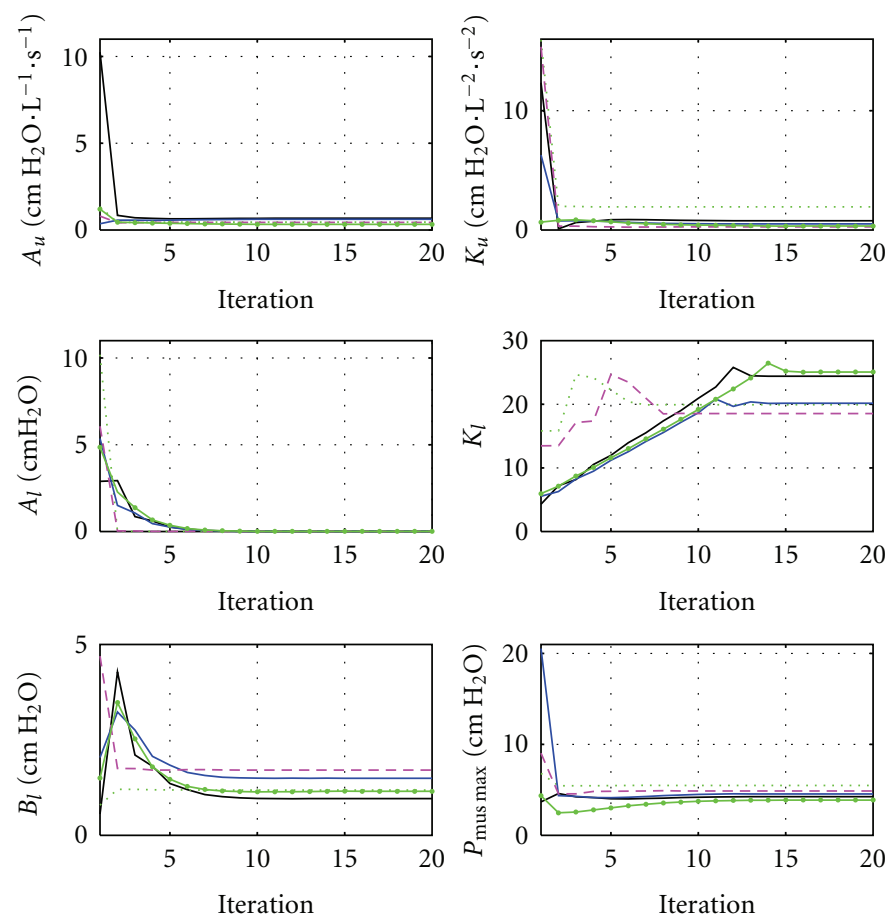

(b)

FIGURE 8: (a) Linear RIC model parameter estimation for five different breath cycles, produced by representative COPD patient's signals. (b) Nonlinear model parameter estimation for five different breath cycles, produced by representative COPD patient's signals.

system (National Instrument DAQCard-6036E ADC-16bit) and stored into the computer for signal processing.

The airway flow signal was the first software filtered to remove high-frequency noise with 8th-order Butterworth low-pass filter with cutoff frequency of $50 \mathrm{~Hz}$, and then processed to detect the breathing cycle onset and end. Recorded signals were divided by breathing cycles with the consideration of the ventilator trigger time, the inspiration, and the expiration time. Five clear breathing cycles were chosen for the processing step. Accordingly, the parameters $T, T_{I}, t_{\text {trig }}$ (i.e., $N, N_{I}$, and $k_{\text {trig }}$ ), PEEP, and $P_{\mathrm{ps}}$ were set to the real values measured from the subject's respiratory signals. $\tau_{\mathrm{vi}}$ and $\tau_{\text {ve }}$ were $0.006 s$ and $\tau_{m}$ was set to $60 \mathrm{~ms}$. MLE algorithm depicted in Algorithm 1 was applied to the measured respiratory signals, and the resulting measurement noise variance, measurement noise shape factor, and parameter convergence tracks were plotted. In order to assess the estimator, results from five different breath cycles were illustrated on the same figure.

\section{Results and Discussion}

5.1. Measurement Noise Variance and Shape Factor Estimation. The variance and the shape factor of the measurement 

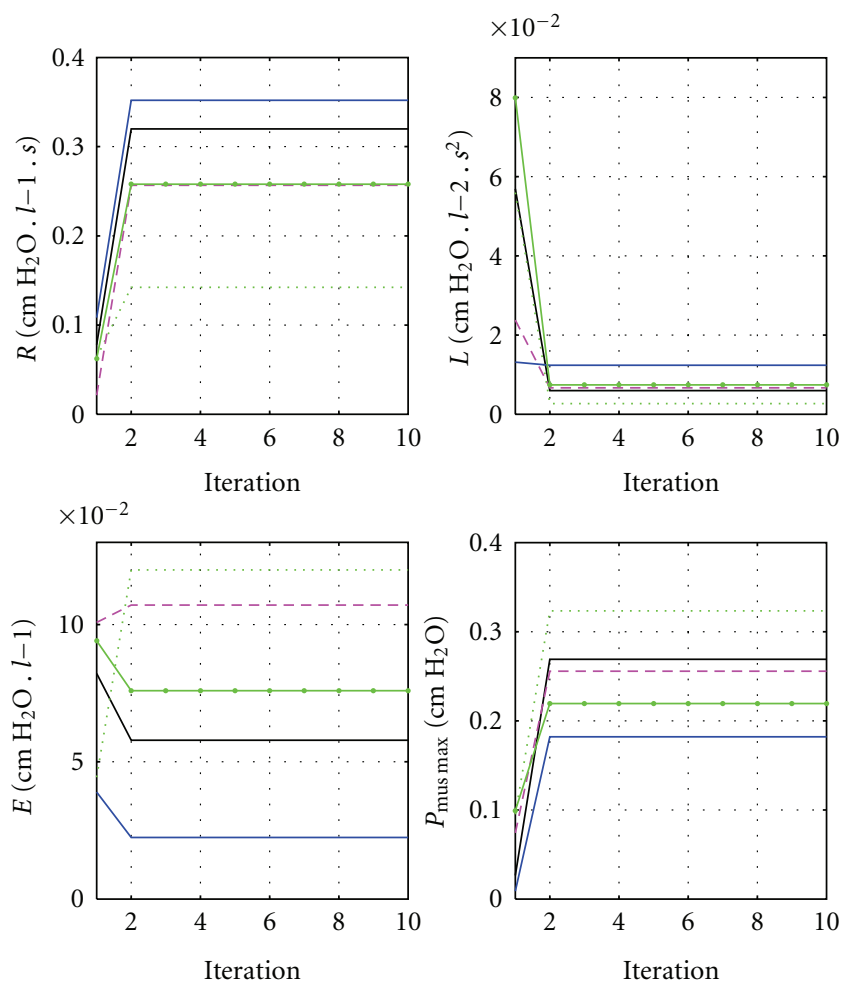

(a)
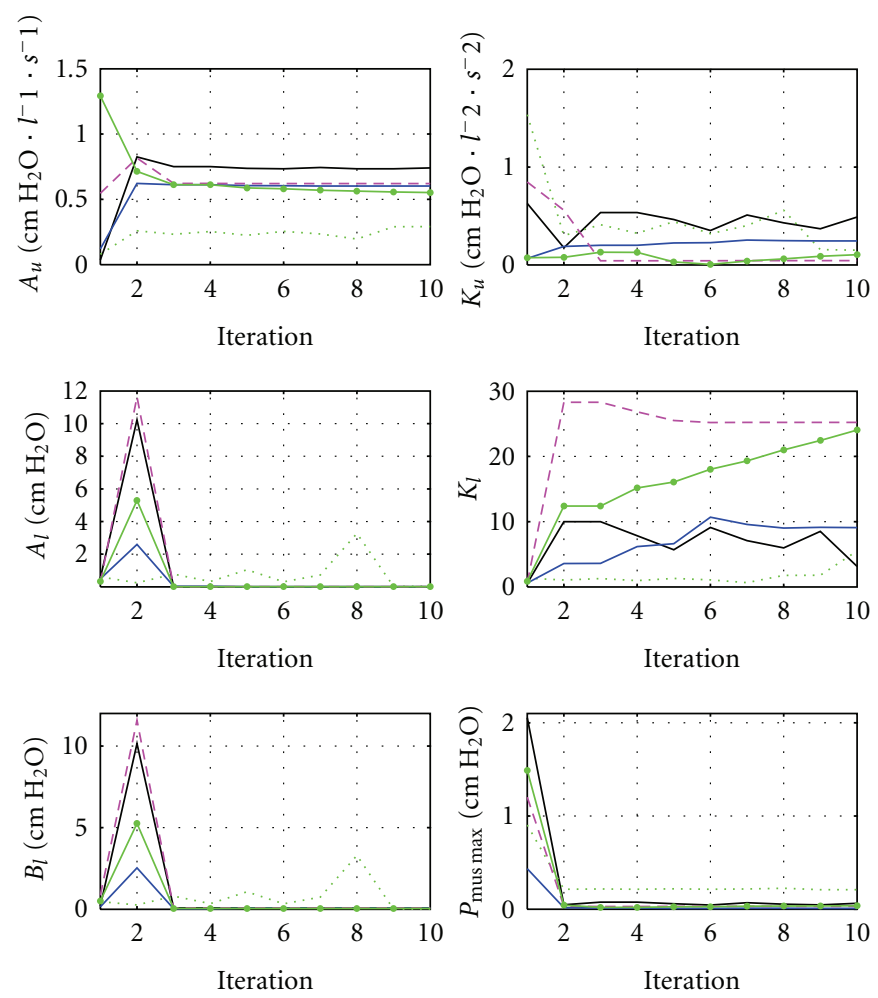

(b)

FIGURE 9: (a) Linear RIC model parameter estimation for five different breath cycles, produced by representative healthy subject's signals. (b) Nonlinear model parameter estimation for five different breath cycles, produced by representative healthy subject's signals.

noise were estimated for all subjects by MLE as depicted in Algorithm 1. Figures 4 and 5 show the estimated $\sigma_{r}^{2}$ for the representative subjects in the Patient group and in the Control group, respectively. Estimated $p_{r}$ is also shown in Figures 6 and 7 for the representative subjects in the Patient group and in the Control group, respectively. First of all, it is apparent that both $\sigma_{r}^{2}$ and $p_{r}$ were converged to the specific value for both groups and for all breath cycles.

From Figures 4 and 5, we can observe that measurement noise variance is much higher in the Patient group than in the Control group. As it is explained in the above section, the initial $\sigma_{r}^{2}$ is set to 10 times higher in the Patient group due to the convergence of the variance, and in the RIC model it is increased to nearly 2 . Also we can see that increase in $\sigma_{r}^{2}$ is higher in the RIC model; thus, for the nonlinear RC model, estimated measurement noise variance is lower in the Patient group. Hence, first important finding can be drawn that Patient group respiratory signals are fitted to the nonlinear RC model with lower measurement noise variance (i.e., lower the variance of the residuals) than to the linear RIC model. Since the lower the variance the better the distribution spotted (which means that the residuals are concentrated to the mean with minimum variance), it is easily concluded that the nonlinear RC model is better fitted to the respiratory signals acquired from Patient group. On the other hand, for the Control group it is totally reverse. Respiratory signals from each healthy subject resulted in higher $\sigma_{r}^{2}$ in the nonlinear RC model, so that the RIC model is better fitted to the respiratory signals from Control group.

We can also support the above argument with the $p_{r}$ estimates. From Figure 6(b) we see that nearly all breaths were converged to $p_{r} \sim 1$, but Figure 6(a) shows more scattered convergence of the waveforms. Control group signals also converge to the specific value for the RIC model, whereas in the nonlinear RC model they were spread in the super-Gaussian area (see Figure 7). Since it is desirable to have concentrated convergence tracks, RIC and nonlinear RC models better describe the respiratory signals from Control and Patient groups, respectively.

Moreover, Figures 6 and 7 show another very important finding: respiratory signals from Patient group resulted in the shape factors in the sub-Gaussian area whereas superGaussian distributed measurement noise was achieved by the Control group respiratory signals. This very distinct result directly indicates the distribution of the measurement noise (i.e., the residuals). The Patient group respiratory signals are fitted to the respiratory models with sub-Gaussian distributed residuals (mostly Laplacian-type $p_{r} \sim 1$ ), whereas super-Gaussian distributed residuals resulted when Control group respiratory signals are processed.

These above findings are the direct consequence of three important effects: (i) the properties of the measured respiratory signals (i.e., airway flow and mask pressure), (ii) the noninvasive ventilator, and (iii) the used respiratory 
system models. The properties of the measured signals are defined as the length of the processed signals (usually very short in the Patient group), energy content of the signal (airway flow is the energy signal in the Control group, whereas for the Patient group it is defined as a power signal), shape of the signal (Control group signals are uniform and smooth signals without sharp edges), and the uncertainty hidden in the signal. Noninvasive ventilator has major contribution to the properties of the respiratory signal, and more importantly respiratory system response is altered with the presence of ventilator-generated pressure. Finally, respiratory models have influence on the result by execution of different mathematical relations. Hence, these items have effects on the results in different scale by comprising the differences between Patient and Control groups and between the RIC and nonlinear RC models.

\subsection{Convergence of Parameters for Measured Respiration Sig-} nals. Convergence waveforms of both RIC and nonlinear RC models' parameters processed for five different breath cycles were given in Figures 8 and 9 for the representative COPD patient's signals and healthy subject's signals, respectively. First of all, it can be observed that, for most of the parameters in different breath cycles, the estimates are converged to the specific value throughout the iterations (notice that $i_{\max }=20$ for Figure 8(b), because of the convergence purposes).

If RIC and nonlinear RC models were compared to each other, we saw that RIC model resulted in very flat estimations (fast convergence). However in the nonlinear model, we have convergence problems at the nonlinear parameters $\left(K_{l}\right.$ and $A_{l}$ ) for the Control group (see Figure 9(b)). It is true, even though we have very smooth $\sigma_{r}^{2}$ and $p_{r}$ estimates. This result encourages the findings that nonlinear RC model is not a good representation of the Control group respiratory signals. On the other hand, it can be easily depicted from Figure 8 that breath-to-breath variations are higher in the RIC model than in the nonlinear RC model for the Patient group. Since it is desirable to have minimum breath-to-breath variations, we again conclude that, for Patient group, nonlinear RC model is better fitted to the measured respiratory signals.

The closer look at Figures 8 and 9 reveals the estimated parameter values. We can see that, for both groups, parameter $R$ in the RIC model and parameter $A_{u}$ in the nonlinear RC model; as well as parameter $P_{\text {mus max }}$ in the RIC and nonlinear RC models were converged to the more or less same values. $P_{\operatorname{mus} \max } \leq 1$ was estimated for the Control group, whereas always $P_{\text {mus max }} \geq 1$ was observed in the Patient group. That shows that the healthy person uses inspiratory muscles less than the COPD patients. We also observed that parameters $R$ and $A_{u}$ are always higher values in the Patient group than in the Control group. Finally, parameter $E$ was converged to values less than 1 in the Control group, whereas it is well above 10 for the Patient group.

5.3. Performance of the MLE for Measured Respiration Signals. The MLE approach and its theoretical performance of the MLE were covered in Section 3.2, and the estimator performance on the parameter convergence was discussed in Section 5.2. However, to be complete, we have to mention that MLE method has potentially serious limitations and constraints in terms of convergence in measured respiratory signal processing. As seen from Figure 8(b) and Figure 9(b), for the nonlinear RC model we have considerable convergence difficulties on nonlinearly related parameters. Moreover, it is observed that MLE required longer computation time for the nonlinear RC model than for the linear RIC model. Performance degradation on MLE can easily be attributed to the nonlinear, non-Gaussian state-space models. The major estimation problem central to gradientbased approaches, such as MLE, is essentially dealing with the initialization. However, as it is covered earlier, confounds between the parameter estimates, including the intraindividual differences, demonstrated the ability of the MLE with the non-Gaussian noise as a diagnostic tool for the COPD patients. Thus, we see these methodological difficulties not so much as a hurdle but more as an even stronger incentive for the biomedical scientists to include intensive repeated researches in nonlinear and non-Gaussian models.

\section{Conclusion}

In this paper we introduced a time-domain methodology to determine the respiratory mechanics of COPD patients under the non-invasive ventilation based on the inverse system modeling approach. Maximum likelihood estimation implemented by Newton-Raphson algorithm yielded optimum estimators for both the linear RIC and the nonlinear RC models. Moreover, the use of generalized Gaussian distribution provided a crucial factor in explaining the measurement noise distribution in the real signal case. We found that respiratory signals from Control group are better represented by the RIC model compared to the nonlinear $\mathrm{RC}$ model. On the other hand, the nonlinear RC model is fitted to respiratory signals from Patient group with lower measurement noise variance, better converged measurement noise shape factor, and model parameter tracks. Also, it is observed that, in the Control group, the shape factor of the measurement noise is much larger than in the COPD patients group. That indicates that the uncertainties between the patients' respiratory signals and the respiratory models have Laplacian distribution, whereas for the Control group it is in the super-Gaussian area.

Finally, MLE is proven to be very powerful statistical signal processing technique for the parameter estimation in the non-Gaussian noise. By using the real respiratory signals, the respiratory system is identified for Patient and Control groups. However, although the algorithm is applied to the dataset obtained in our labs, the validation with more datasets and subjects will confirm the applicability of the MLE algorithm.

\section{Acknowledgments}

The authors gratefully acknowledge the contribution of Prof. Dr. Ertugrul Eris for the technical support, Prof. Dr. 
Nurhayat Yildirim for the COPD patients' data acquisition, and reviewers' comment. This paper was partially supported by The Research Fund of The University of Istanbul. Project nos. are 3898, BYP-6687, and T-965/06102006.

\section{References}

[1] J. M. B. Hughes and M. B. Pride, Lung Function Tests: Physiological Principles and Clinical Applications, W. B. Saunders, London, UK, 2000.

[2] A. G. Polak and J. Mroczka, "Nonlinear model for mechanical ventilation of human lungs," Computers in Biology and Medicine, vol. 36, no. 1, pp. 41-58, 2006.

[3] A. Athanasiades, F. Ghorbel, J. W. Clark Jr. et al., "Energy analysis of a nonlinear model of the normal human lung," Journal of Biological Systems, vol. 8, no. 2, pp. 115-139, 2000.

[4] A. Eberhard, P.-Y. Carry, J.-P. Perdrix, J.-M. Fargnoli, L. Biot, and P. F. Baconnier, "A program based on a 'selective' least-squares method for respiratory mechanics monitoring in ventilated patients," Computer Methods and Programs in Biomedicine, vol. 71, no. 1, pp. 39-61, 2003.

[5] B. Diong, H. Nazeran, P. Nava, and M. Goldman, "Modeling human respiratory impedance," IEEE Engineering in Medicine and Biology Magazine, vol. 26, no. 1, pp. 48-55, 2007.

[6] J. H. T. Bates and A.-M. Lauzon, "A nonstatistical approach to estimating confidence intervals about model parameters: application to respiratory mechanics," IEEE Transactions on Biomedical Engineering, vol. 39, no. 1, pp. 94-100, 1992.

[7] B. Suki, H. Yuan, Q. Zhang, and K. R. Lutchen, "Partitioning of lung tissue response and inhomogeneous airway constriction at the airway opening," Journal of Applied Physiology, vol. 82, no. 4, pp. 1349-1359, 1997.

[8] E. Saatçi and A. Akan, "Lung model parameter estimation by unscented Kalman filter," in Proceedings of the Annual International Conference of the IEEE Engineering in Medicine and Biology Society, pp. 2556-2559, 2007.

[9] Y. Yamada and H.-L. Du, "Analysis of the mechanisms of expiratory asynchrony in pressure support ventilation: a mathematical approach," Journal of Applied Physiology, vol. 88, no. 6, pp. 2143-2150, 2000.

[10] K. Kokkinakis and A. K. Nandi, "Exponent parameter estimation for generalized Gaussian probability density functions with application to speech modeling," Signal Processing, vol. 85, no. 9, pp. 1852-1858, 2005.

[11] S. M. Kay, Fundamentals of Statistical Signal Processing, Prentice-Hall, Englewood Cliffs, NJ, USA, 1993.

[12] T. T. Pham and R. J. P. DeFigueiredo, "Maximum likelihood estimation of a class of non-Gaussian densities with application to Lp deconvolution," IEEE Transactions on Acoustics, Speech, and Signal Processing, vol. 37, no. 1, pp. 73-82, 1989.

[13] B. T. Sieskul and S. Jitapunkul, "An asymptotic maximum likelihood for estimating the nominal angle of a spatially distributed source," International Journal of Electronics and Communications, vol. 60, no. 4, pp. 279-289, 2006. 